\title{
Airfoil LineAR Wind GENERATOR (ALWG): As A NOVEL WIND ENERGY EXTRACTION APPROACH
}

\author{
Hossein Darijani ${ }^{1}$ and Abbas Panahi ${ }^{2}$ \\ ${ }^{1,2}$ Department of Mechanical Engineering, College of Engineering, Shahid \\ Bahonar University of Kerman, Kerman, Iran
}

\begin{abstract}
Linear wind generator ( $L W G)$ is a sufficient way of wind energy harnessing process. However, complicated $L W G$ energy extraction mechanism such as complex system for transferring linear motion to rotational motion and problems related to changing the angle of attack is resulted to energy dissipation. In the other hand the linear generator that delivers ocean wave energy to electricity has been developed as a new renewable energy extraction method. Some of the problems associated with this technology are corrosion, high cost of manufacturing, high requirement for installation and construction, economical consideration, etc. In the most recent works, low dissipation energy in mechanism, low cost, simplicity and high performance are highly regarded as environmentally friendly methods for wind energy extraction mechanisms. In the current study, we would like to introduce a new and efficient method to extract wind energy using airfoil linear wind generator(ALWG). ALWG is a new method that produces liner reciprocating motion via attached airfoils to a mover in a magnetic field in order to generate electricity. The most important advantage of $A L W G$ is its simplicity and its compatibility to all wind situations that can be more controllable relative to ocean-based and also relative to $L W G$ that become challengeable problem.
\end{abstract}

\section{KEYWORDS}

Keywords: ALWG, Airfoil, Linear Generator, Wind Energy

\section{INTRODUCTION}

An urgent and strategic issue of our society is the solution of the atmosphere pollution problems which posed by electric energy generation from fossil flues as nonrenewable sources [1]. Obviously, these problems can be resolved by the use of natural sources that are renewable, cheap, easily available and sustainable for the environment. However, the actual renewable technologies up to now had not such potentials. As a result, many attempts have been conducted in order to utilize wind [2], ocean wave [3], solar [4], etc. as green energy sources. One of the most well-known examples is wind mills or wind turbine that is used as a realistic approach to harness wind energy in great amount. Wind farms have problems of land occupation and environmental impact because of this fact that their electrical production depend on the amount of area that they occupy [5]. To overcome these problems, Milanese et al. has developed a new class of wind energy generators, named "Kitegen" as shown in Figure 1, [6, 7]. 


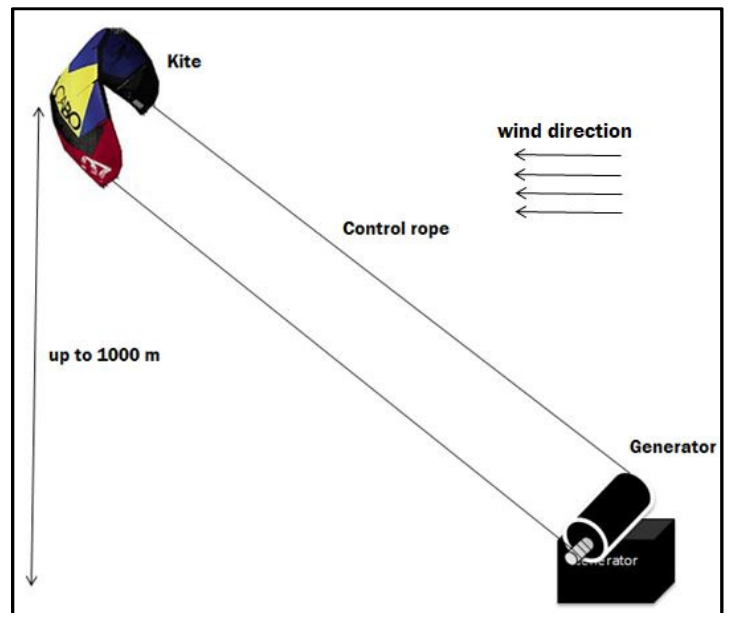

Figure 1. The mechanism of operation of kite generator

There are many obstacles in front of Kitegen project development, due to the main problems that wind has created for controlling the trajectory of kite and its stability [8]. Up to now, there are rare methods to utilize this project efficiently. Consequently, there is much consideration on Kitegen project in the recent years in order to extract wind energy using this method practically. For example, Laddermill [9] with similar situations to Kitegen is one of the used technologies. In the Laddermill structure there are many kites which are connected to a main rode that is twisted around an attached drum to generator (Figure 2).

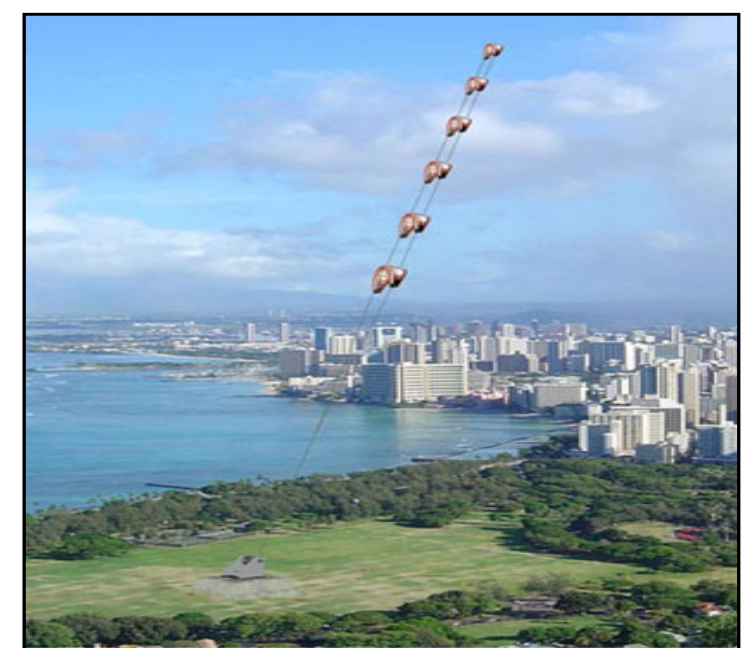

Figure 2. The structure of a Laddermill

Another important approach which has been introduced based on Kitegen operation principle, is linear wind generator (LWG). The LWG can convert an equivalent swept area of wind compared to wind turbine generator (WTG) into twice the amount of electricity. Also, it is simple to install and can produce electricity at a substantially lower cost and within a significantly smaller land area compared to WTGs.

To introduce the LWG operation its principle is demonstrated in Figure 3. 


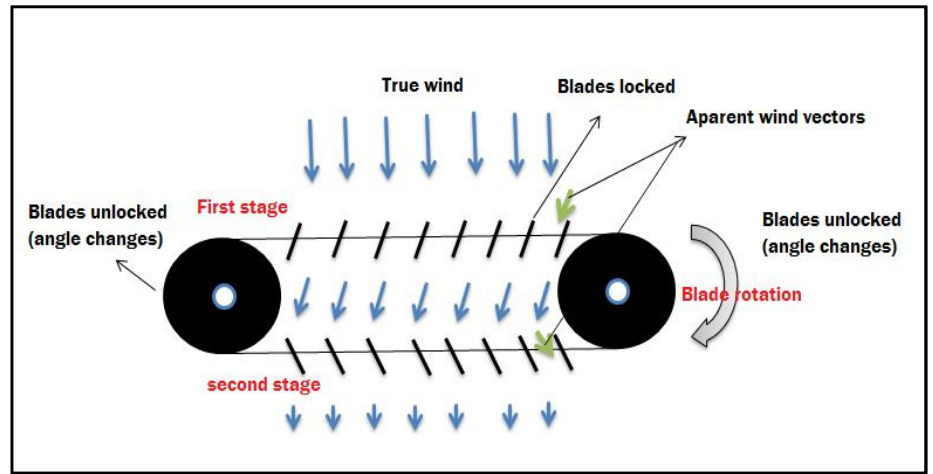

Figure 3. The principle of action of a LWG

As shown in Fig.3, first, air passing over the initial stage blades to produce a lift force. The air velocity, after passing the first stage, is reduced significantly and requires the second stage blades to be angled appropriately because of the changed apparent wind vector. The second stage blades also produce a lift force component in the direction of cable travel thereby adding to the first stage's contribution; by this way the most amount of winds energy have been harnessed .

As you can see in the LWG operation mechanism, it suffers from a complicated energy extraction process due to a complex system for transferring linear motion to rotational motion. Furthermore, it has problems originated from changing the angle of attack which are resulted to the energy dissipation and system life reduction.

One of the newest ideas is the use of linear generator (LG) to convert ocean wave energy to electricity [10]. The simple structure of a LG for energy extraction from ocean is shown in Figure 4.

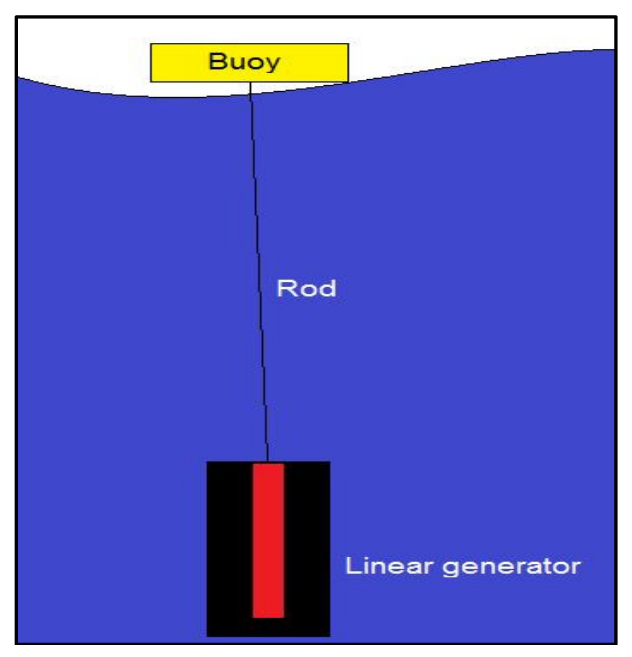

Figure 4. The main structure of an ocean wave energy generator

Although the energy extraction from ocean ,[11], is interesting and can be used for special applications, however it suffer from some drawbacks such as corrosion, high cost of manufacturing, high requirement for installation and construction, economical consideration, etc. [12] . Nowadays, some modifications to already existing methodologies to renewable energy extraction, considering the environmental consciousness in both industrial and academic studies, have received great attention. Along this line, herein, we introduce a new mechanism (Figure 5) 
based on aerodynamic forces exerts on airfoil by air current. In this method we used from linear generator instead of rotary generator. Overall, in our protocol the lock-unlock mechanisms and angle of attack changer is eliminated in order to save energy by use of a flywheel and change of angleof attack only.

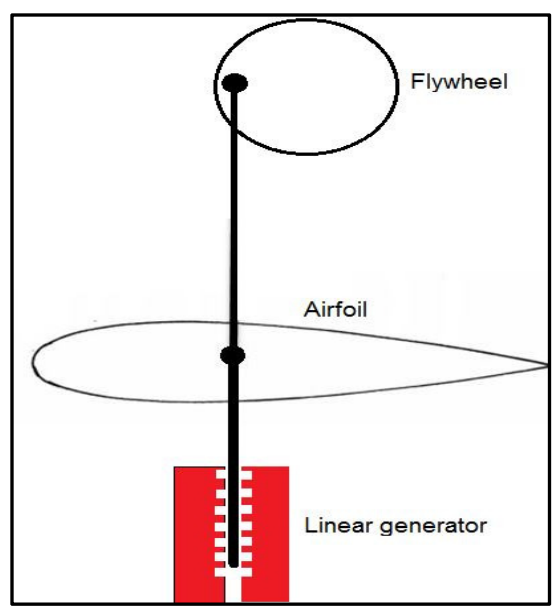

Figure 5. Overall picture of an ALWG

\subsection{The structure and mechanism of AWLG}

The main structure of a designed AWLG is depicted in Figure 6.

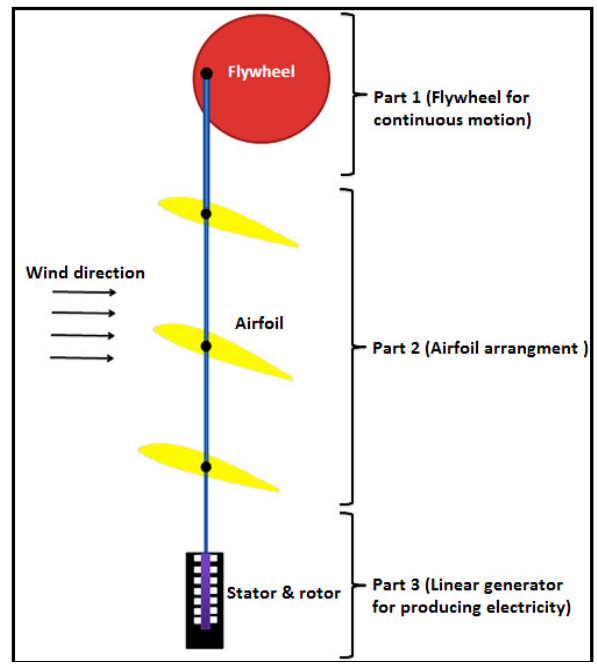

Figure 6. The main components of ALWG and its operation

As shown in Fig. 6, an AWLG contains 3 substructures: 1) a set of airfoils, 2) linear generator and 3 ) a device for saving energy (flywheel or spring). In AWLG, in first cycle the angle of attack of airfoil will be changed in order to exert an upward force on airfoil. While the airfoil reaches to the maximum level in the vertical path, simultaneously the flywheel is saving fraction of the energy. After this process the flywheel operates and complete the other cycle. Taking into account the fact that airfoil is on a beam, a reciprocating motion will be produce in perpendicular direction relative to wind. Considering this issue that radial and axial force occur in the linear generator (between rotor and stator, this force is due to the geometrical magnetic anisotropy of the 
generator), the existence of flywheel is necessary in order to maintain continues motion and homogenize anisotropy forces [13]. In the second cycle (operation of flywheel), the changer of angle of attack mechanism can deplete it to minimum level. This action decrease the lift force on the airfoil to zero as it reaches to minimum length relative to generator. Affected by this actionthe flux in the stator coils varies with the mover position from maximum to minimum. Consequently, an $\mathrm{AC}$ motion-induced voltage is produced in the stator coil as a result of up-and-down oscillatory motion of the mover.

\subsection{Conservation of energy}

The magnetic flux $(\varphi)$ can be determined using the following equation (Eq. 1) in the presence of a magnetic field (B):

$\emptyset=\int B \cdot d \vec{a}$

Also, to determine the electromotance, in the presence of a magnetic field the Faraday induction low is used (Eq. 2). (For more study [14])

$e=-\frac{d(N \varnothing)}{d t}$

According to this equation the electromotance (voltage) induced in a close circuit are equal minus the time derivation of total flux enclosed by the circuit. The minus sign is confirmed that the direction of the current induced in the circuit is opposed to the direction of increasing flux.

The mechanism of operation of linear actuator according to the equations 1 and 2 is shown in Figure 7, which is the principle of a linear generator in reverse manner.

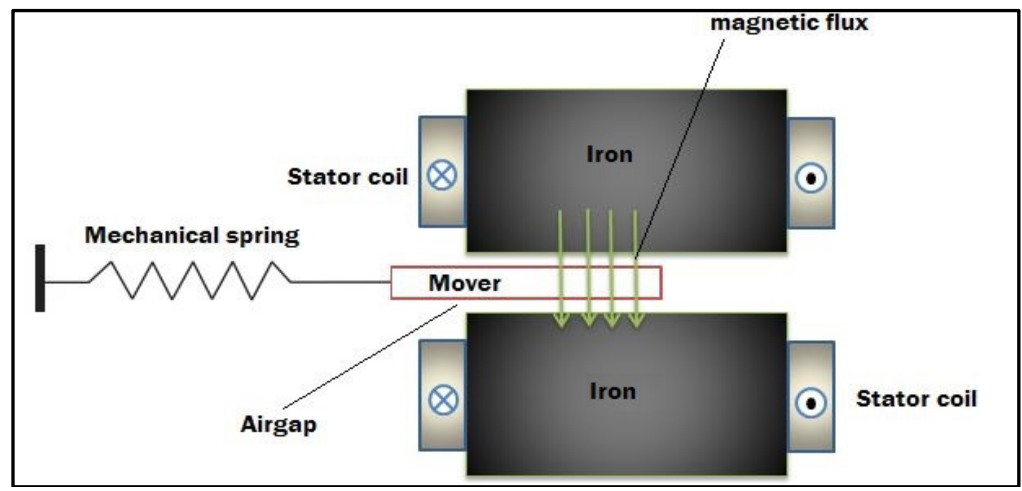

Figure 7. The principle of operation of linear generator \& actuator

Because the electromechanical energy conversion process is reversible, actuators can be operated as linear electric generators, in which case mechanical energy is transformed into electric energy. The fundamental energy conversion equation is demonstrated in Eq. 3.

$$
F \dot{x}=V I
$$

Where $F$ is mechanical force, $\mathrm{N} ; \mathrm{dx} / \mathrm{dt}$ is mechanical velocity $\mathrm{m} / \mathrm{s} ; \mathrm{V}$ is voltage, and I is current. It is assumed that $F$ and $x$ are in the same direction. 
When the stator coil is fed with an electric current, the ferromagnetic translator is pulled into the air gap (between the stator poles) until, for $x=0$, the force becomes zero. At $x=0$ all the flux lines ideally become vertical (aligned flux position) and the stored energy in the magnetic field is minimal. Now, if the current is turned off, the mechanical spring pulls the translator out from the air gap to $x=-l / 2$ position. At this position the current is turned on again. Sustained oscillations obtain. This mechanism explain the work of linear actuator ,but it is practical that we reverse the mechanism in order to getting electricity by enforce mover to experience a reciprocating motion .We do this work by means of lift force that has been accomplished by an airfoil [14] .

\subsection{Mathematical modeling of motion of airfoil}

Figure 8 schematically shows an airfoil installed in wind direction.

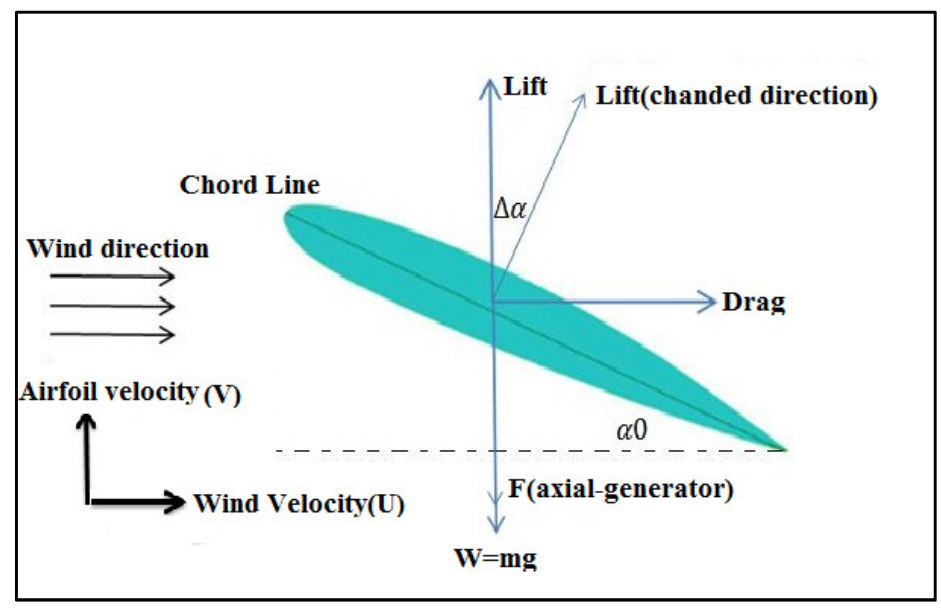

Figure 8. The mathematical modeling of an airfoil motion

It is assumed that the airfoil can move only vertically. The displacement of the center of mass is $\mathrm{z}$ and it is positive when going upward. The wind is blowing at uniform horizontal of speed $u$. When the airfoil moves upward at a speed of $\mathrm{v}$ the surrounding air moves downward relative to the airfoil at the same speed. To the oncoming air flow, the angle of attack of the airfoil can be obtained by use of the following equation:

$$
\alpha=\alpha_{0}+\tan ^{-1}\left(\frac{v}{u}\right)
$$

In absence of wind the angle of attack of airfoil is $\alpha_{0}$ and after that airfoil senses wind the angle of attack increases as amount of $\tan ^{-1}\left(\frac{v}{u}\right)$.

The lift of the airfoil in the direction normal to the flow has the expression as:

$$
L=\frac{1}{2} \rho\left(u^{2}+v^{2}\right) S C l
$$

Where $\rho$ is air density, $\mathrm{S}$ the projected area of airfoil, and $\mathrm{C}_{\mathrm{L}}$ is the lift coefficient of the airfoil. The lift coefficient increases by enhancement of the angle of attack up to stall point. It should be mentioned that the angle of attack will be reduced, if the rising of angle of attack continue. The curve of $C_{L}$ versus angle of attack is shown in Figure 9. 


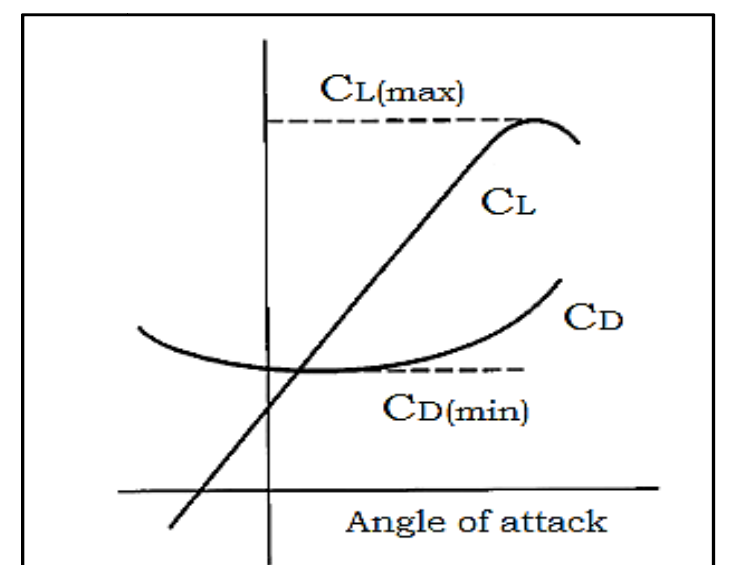

Figure 9. Lift coefficient $\left(C_{L}\right)$ versus angle of attack $(\alpha)$

In this study the thin airfoil theory is used in which lift coefficient has a linear relation with angle of attack (Eq. 6).

$$
C_{L}=2 \pi \alpha
$$

It is necessary to avoid very high angle of attack due to the effect of induced drag that cause exerting an undesirable force in direction of air flow. In high angle of attack, the linear generator may experience unfavorable input and this can effects on beam that airfoil has a reciprocating motion on it (Fig. 5). Consequently, $\alpha$ should be selected in a certain range in order to avoid stall. More importantly, 'vortex' is an imperative point that could exist when aspect ratio (AR) is finite. Vortex causes some circulation behind of the airfoil, unbalanced pressure distribution on top and below of the airfoil, that all together cause low effective angle of attack relative to airfoil section with infinite aspect ratio $(\mathrm{AR}=\infty)$. In this work for simplicity we ignored the effects of drag force and induced drag as two main factors in structure designing.

The equations of motion for the center of mass are:

$\frac{d z}{d t}=v \quad$ and $\quad m \frac{d v}{d t}=-m g+L \cos (\Delta \alpha)-f_{\text {axial }}$

Upon substitution of the value $\cos \Delta \alpha=\frac{u}{\sqrt{u^{2}+v^{2}}}$ and $\mathrm{L}$, the second equation becomes:

$m \frac{d v}{d t}=-m g-f_{\text {axial }}+\pi \rho S u\left(\alpha_{0}+\tan ^{-1}\left(\frac{v}{u}\right)\right) \sqrt{u^{2}+v^{2}}$

In which $\mathrm{m}$ is the sum of mass of airfoil and rotor ( considered $5 \mathrm{Kg}$ ), $\mathrm{u}$ is velocity of wind, $v$ is velocity of mover, $f_{\text {axial }}$ is axial force due to the effect of geometrical magnetic anisotropy of the generator , $\alpha_{0}$ is initial angle of attack , $\mathrm{S}$ is projected area of airfoil .

By considering the fact that electrical power plus losses in generator due to hysteresis, iron, eddy current... etc. is equal the mechanical power, so we determined its value as follow:

$$
P=F V
$$


By substituting (8) into (9) following equation will be obtained:

$$
P=\left(-m g-f_{\text {axial }}+\pi \rho S u\left(\alpha_{0}+\tan ^{-1}\left(\frac{v}{u}\right)\right) \sqrt{u^{2}+v^{2}}\right) v
$$

And also eq. 11 is as follow:

$$
P=\frac{V^{2}}{R}
$$

In Eq. $11 \mathrm{R}$ is the equivalent resistant in circuit which depends on the generator and also electrical properties of stator and rotor which is considered its value a constant. Figure (10) shows schematically a lumped circuit equivalent of the generator can be used to understand how the current in generator is related to induced electromotance.

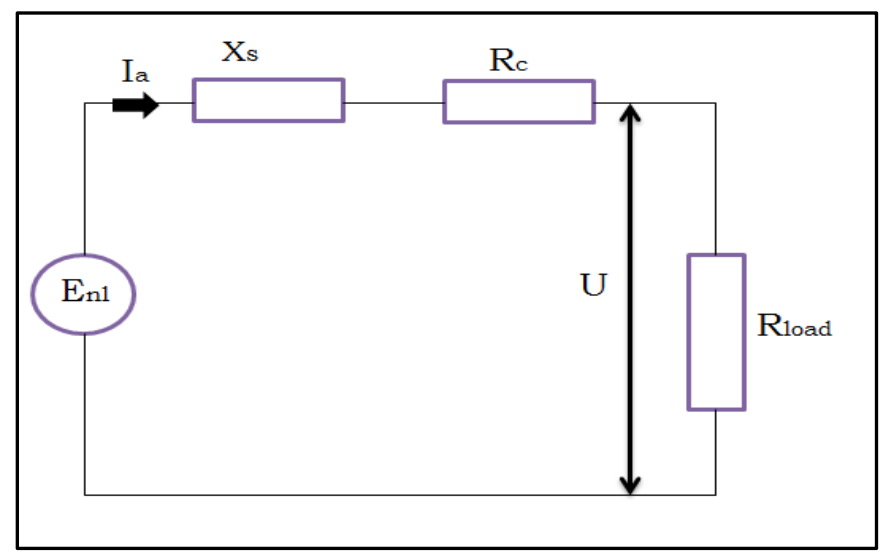

Figure 10 : Lumped circuit equivalent of a synchronous generator

The circuit equivalent is only an approximation but is useful for pedagogical reasons. Figure (10) shows the equivalence of a generator where the measurable voltage is described by an electromotance $\mathrm{E}_{\mathrm{nl}}$. This voltage is referred to no load voltage, which is the measurable voltage at the coil ends when no current flows through the generator's is the reactance of the generator .Rc is the resistance in the coil windings . The outer circuit, or the load, can be either purely resistive or reactive. In this work the load is assumed to be strictly resistive and is described by a resistance $R_{\text {load }}$ The voltage over the load $U$ will thus be in phase with the armature current $I_{a}$.

\subsection{Power losses}

The power losses in a generator consist of three parts:

1. Losses due to the changing magnetic field (often referred to steel losses)

2. Resistive losses in the coil windings

3. Mechanical losses such as friction and deformation.

The main part of these losses happen in the stator steel .It consists of two parts: hysteresis losses and losses due to eddy currents. 


\subsubsection{Hysteresis losses}

Hysteresis losses is the energy it takes to reverse the magnetization of the material and will only effect materials that are magnetic dipoles .The losses are approximately proportional to the frequency of the magnetic field and the acquirer of the magnetic induction and per unit volume is as follows:

$$
P_{h}=C_{h} B^{2} f
$$

Where $\mathrm{C}_{\mathrm{h}}$ is a material dependent constant. Hysteresis losses can be reduced by selecting a material with soft magnetic properties i.e. a material that is easily re-magnetized hysteresis.

\subsubsection{Eddy current losses}

Eddy currents are circular electric current in a material by the changing magnetic field. These current are directly proportional to the electromotance induced in circuit by changing magnetic flux. And the power losses per unit volume is proportional to the square of current as follow:

$P_{E}=C_{E} \frac{1}{T} \int_{0}^{T}\left(\frac{\partial B}{\partial t}\right)^{2} \approx C_{E} B^{2} f^{2}$

$\mathrm{C}_{\mathrm{E}}$ is a material constant depending on the resistivity and $\mathrm{T}$ is the time of one period .The major part of eddy losses appear in stator and can be depleted by assembling them with electric isolated lamination of the stator steel.

\subsubsection{Resistive losses}

The resistive losses in the coil windings are referred to as copper losses because the conductor in most cases are made of copper. Since copper is not a magnetic dipole material hysteresis losses will not appear. The resistive losses in a conductor with electrical resistance R caring current I are:

$$
P_{c}=I^{2} R
$$

Table 1. The value of wasted powers in a considered linear generator

\begin{tabular}{|l|l|}
\hline Hysteresis $(\mathrm{KW})$ & 0.52 \\
\hline Eddy current losses $(\mathrm{KW})$ & 0.07 \\
\hline Rotational losses $(\mathrm{KW})$ & 0.03 \\
\hline Total iron losses $(\mathrm{KW})$ & 0.62 \\
\hline
\end{tabular}

In the Eq.15 the principle of conservation of energy is mentioned, that explain the relation between electrical energy and mechanical energy and also their relevant losses.

$\mathrm{P}($ mechanical $)+\mathrm{P}($ friction-loss $)=\mathrm{P}($ electrical $)+\mathrm{P}($ Copper, iron, ... losses $)$

By substituting (eq. 10) into (eq. 11) and by use of (eq. 15) and also ignoring the amount of losses (because estimating the maximum energy output of this mechanism is regarded). As a result the following equation is obtained. 


$$
V=\sqrt{R_{\text {eqal }}\left(-m g-f_{\text {axial }}+\pi \rho S u\left(\alpha_{0}+\tan ^{-1}\left(\frac{v}{u}\right)\right) \sqrt{u^{2}+v^{2}}\right) v}
$$

After plotting $\mathrm{P}$ (power) versus mover velocity, this shows that mechanical power increase dramatically as velocity increases slightly (Figure 11).

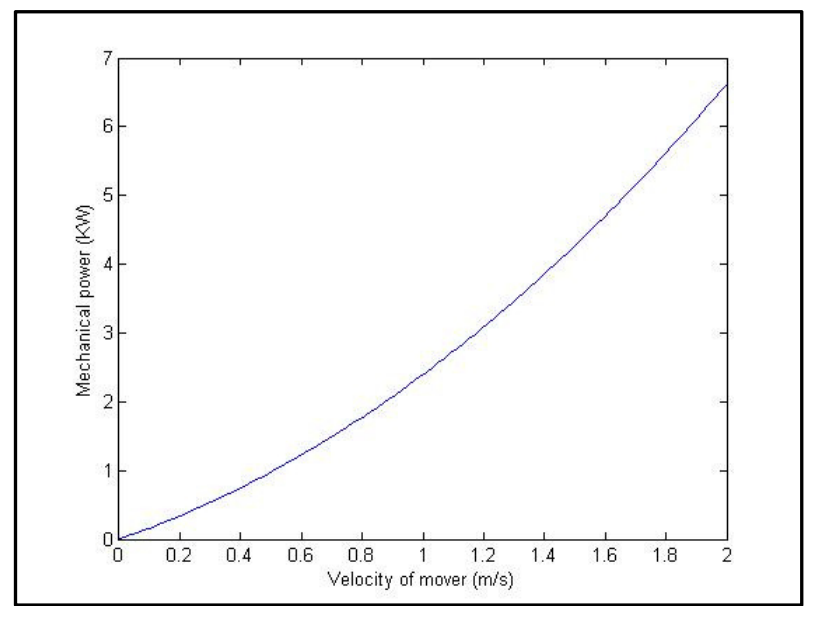

Figure 11: The increment of pure mechanical power versus velocity of mover

It is possible to use several airfoils vertically but what is important here is the distance between the used airfoils. From aerodynamic point of view when air passes on the airfoil, it changes the stream shape and makes a disturbance in the vicinity around airfoil. This function affects the pressure distribution around upper or lower airfoil and generally causes depletion in lift force of them. We supposed that there is no limitation on installing airfoils which have been arranged in a logical manner that causes no bad effect on the other airfoil. By this way the mechanical power that has extracted increase dramatically.

Equation 16 is a relation that shows Induced-voltage versus velocity of mover. It should be considered that velocity can be instantaneous or constant. In the following the induced voltage and mechanical power for some condition are illustrated.

\section{Results and discussions}

The mechanical power was plotted versus velocity of mover as depicted in Figure 12. 


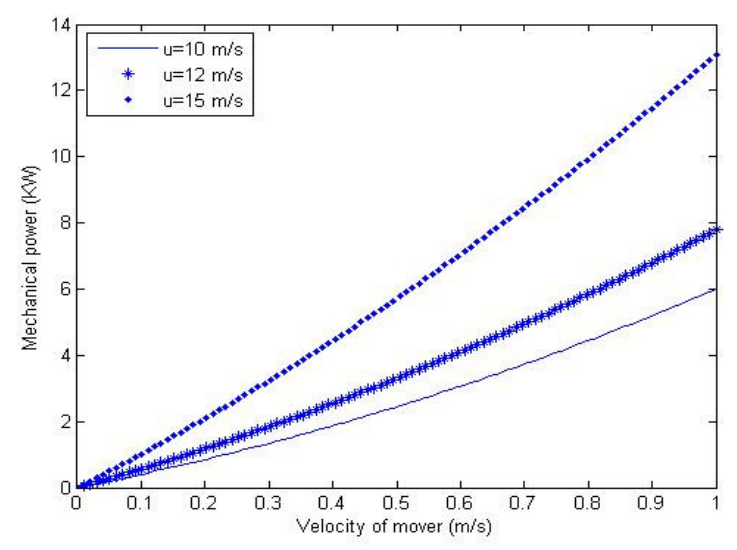

Figure 12. Power versus mover velocity in different wind condition

As shown in Fig. 12 by increasing the wind velocity the amount of mechanical power is increased dramatically.

In other attempt, we investigate the effect of projected area on mechanical power for $u=10 \mathrm{~m} / \mathrm{s}$. The diagram of this investigation is shown in Figure 13.

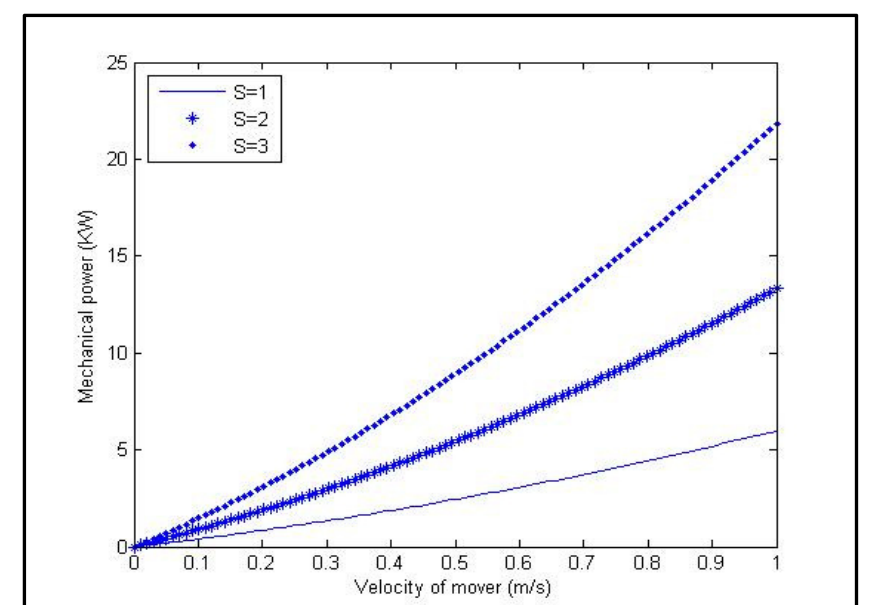

Figure 13. Power versus mover velocity in different value of projected area

Figure 12 show that by increasing the projected area (S) the mechanical power is increased. You can see that the effect of this parameter is more than wind velocity so that in same velocity of mover the power output become 3 times greater than the its amount in figure 12 .

Other important factor to increase the mechanical power is initial angle of attack. As shown in Figure 14 the mechanical power is increased with addition of initial angle of attack. For example for $1 \mathrm{~m} / \mathrm{s}$ velocity of mover, $10 \mathrm{~m} / \mathrm{s}$ velocity of wind and initial angle of attacks 10,14 and 16 the mechanical power were calculated 6,7 and $8 \mathrm{KW}$ respectively. 
International Journal of Recent advances in Mechanical Engineering (IJMECH) Vol.3, No.4, November 2014

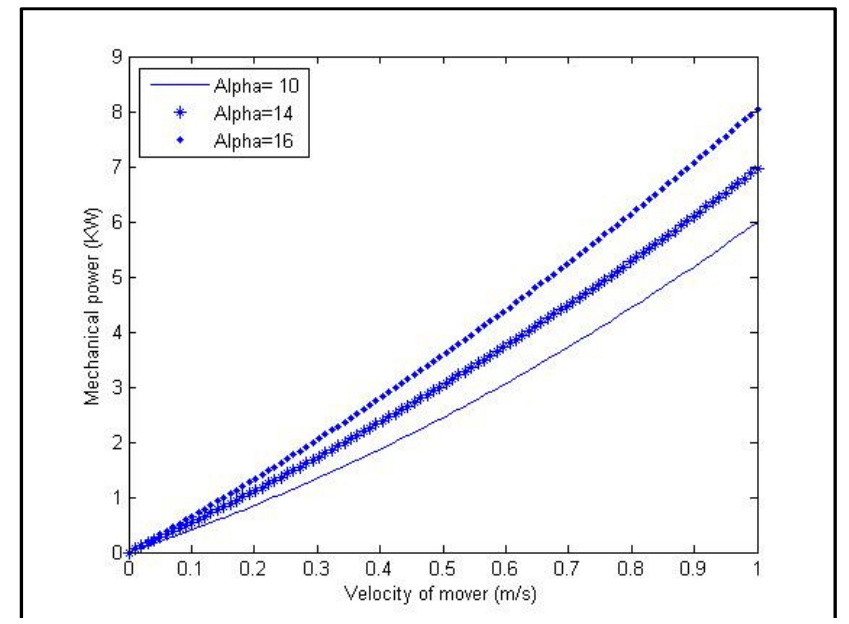

Figure 14. Power versus mover velocity in different initial angle of attack

Our studies on the effect of number of airfoil on mechanical power showed that this parameter has significant influence on power output.

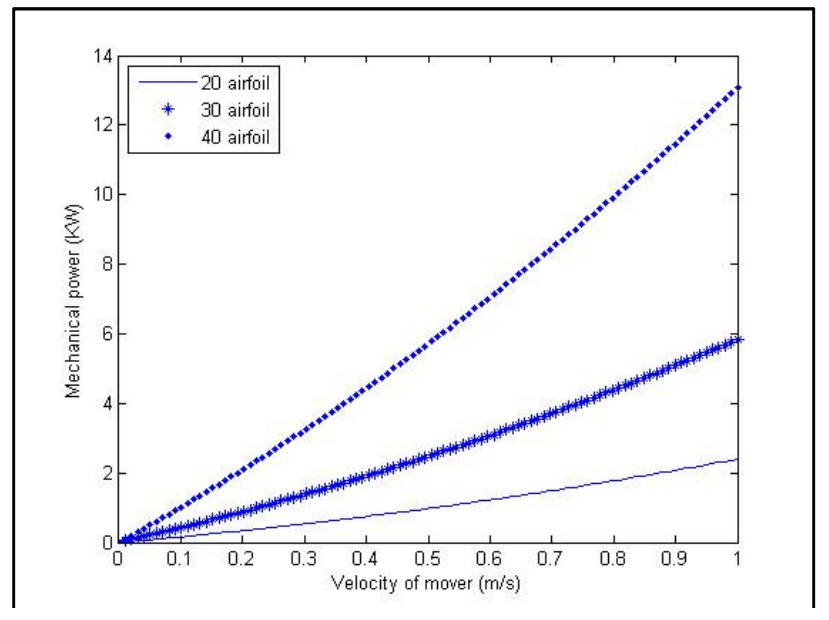

Figure 14. Power versus mover velocity for 20, 30 and 40 number of airfoils

As shown in Figure 14, at a certain velocity of mover when the numbers of airfoils increase twice the power become 7 times approximately. This is very interesting point because we can improve the power output in huge amount using only manipulation the number of airfoils.

Next we evaluated the effect of wind velocity, projection area, and initial angle of attack on the overall induced-voltage in the main circuit of system. The results demonstrated that by increment of wind velocity the output voltage enhance (Figure 15). 
International Journal of Recent advances in Mechanical Engineering (IJMECH) Vol.3, No.4, November 2014

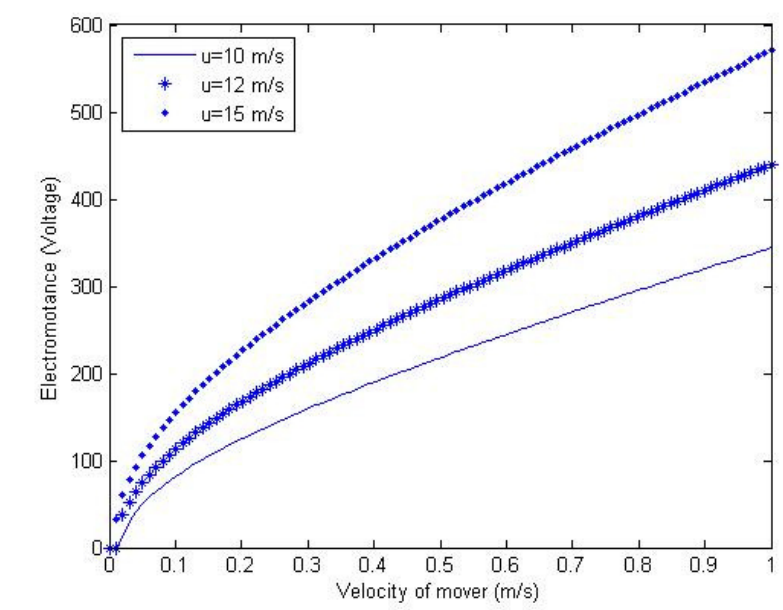

Figure 15. Induced-voltage versus mover velocity in different wind condition

As shown in Figure 15, when the wind velocity changed from $10 \mathrm{~m} / \mathrm{s}$ to $15 \mathrm{~m} / \mathrm{s}$ the voltage become twice. Effect of projected area on voltage is shown in Figure 16.

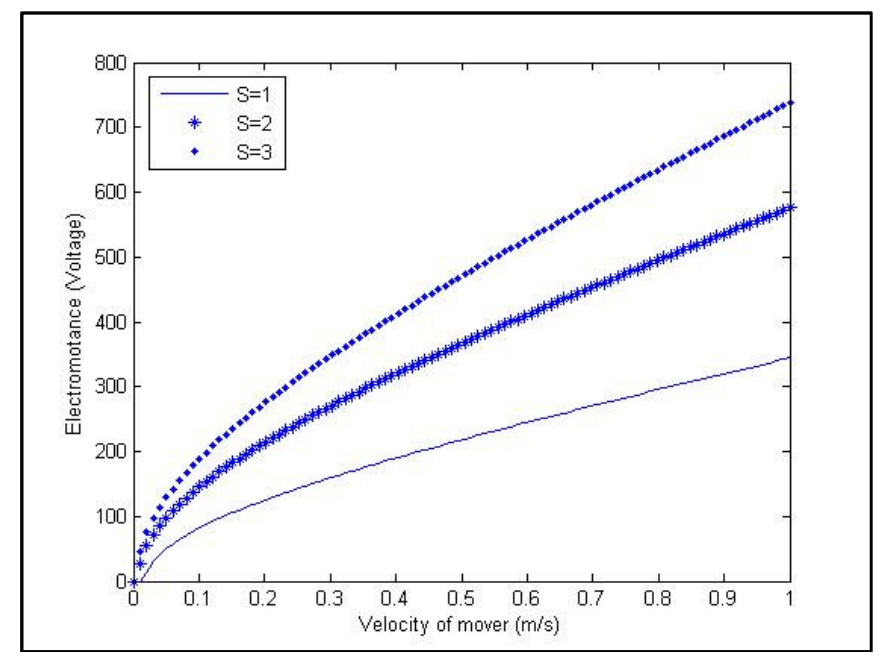

Figure 16. Induced-voltage versus mover velocity in different projected area

Figure 16 shows that by increase of projection area the voltage is increased so that at $u=10 \mathrm{~m} / \mathrm{s}$ when $S$ raise from 1 to 3 voltage change from $350 \mathrm{v}$ to $750 \mathrm{v}$.

Also by increment of initial angle of attack the overall induced-voltage is increased. As shown in Fig. 17, by enhancement of alpha from 10 to 16 at a specific wind velocity the output voltage becomes twice approximately. 


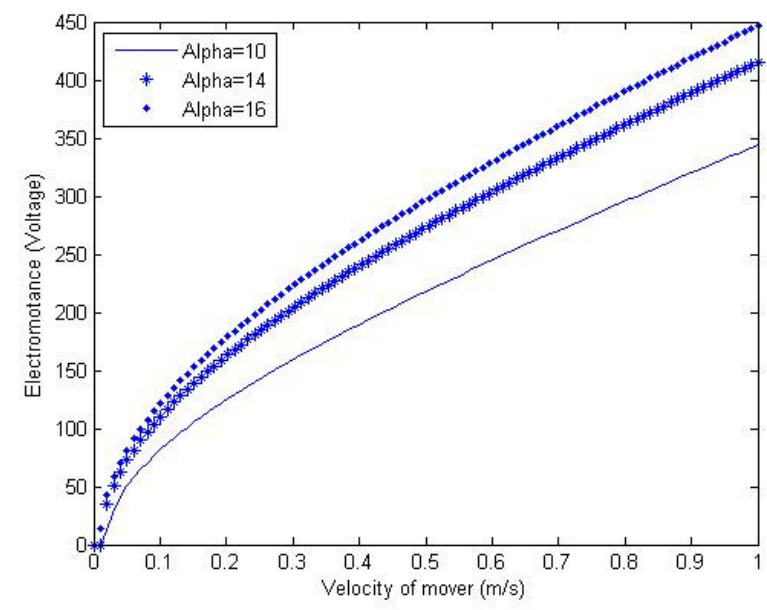

Figure 17. Induced-voltage versus mover velocity for different initial angle of attack

Furthermore, Figure 18 shows the value of mechanical power and velocity of mover in same time, confirming that with past of time these parameters enhance in same manner. This diagram demonstrated that with increment of time that is same to increase the amplitude of oscillation of mover, the power output will increase too.

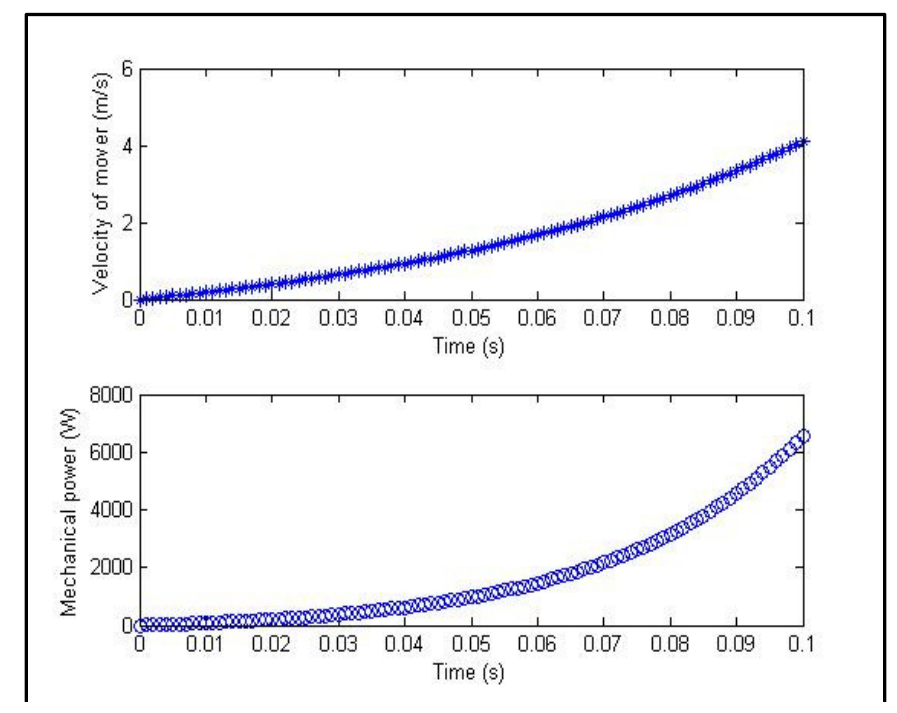

Figure 18 .Value of mechanical power and velocity of mover in same time

\subsection{A comparison between ALWG and ocean wave energy}

The total energy in an ocean wave can be calculated in joules per unit of width of wave front by summing up the potential and kinetic energy of all waves together [15]. The potential energy in a wave of length $L$ is generated by the displacement of the water away from the mean sea level. The kinetic energy of a wave is a result of both horizontal and vertical water particle motions [16]. The total potential and kinetic energy of an ocean wave can be expressed as: 
$E=\frac{1}{2} \rho g A^{2}$

Where $\mathrm{g}=9.8 \mathrm{~m} / \mathrm{s}^{2}$ and $\rho$ is density of water $\left(1000 \mathrm{Kg} / \mathrm{m}^{3}\right)$ and $\mathrm{A}$ is wave amplitude. To obtain the average energy flux or power of a wave period, energy is multiplied by the speed of wave propagation, $\mathrm{V}_{\mathrm{p}}$ :

$V p=\frac{L}{2 T}$

Where $\mathrm{T}$ is the wave period (s) and $\mathrm{L}$ is wavelength (m) [18]:

$P w=\frac{1}{2} \rho g A^{2} \frac{L}{2 T}$

And by following relation $\mathrm{L}$ and $\mathrm{T}$ are related:

$L=\frac{g T^{2}}{2 \pi}$

So the final relation will be:

$P w=\frac{\rho g^{2} T A^{2}}{8 \pi}=\frac{\rho g^{2} T H^{2}}{32 \pi}$

Where $\mathrm{H}$ is the height of wave and $\mathrm{A}$ is amplitude $(\mathrm{H}=2 \mathrm{~A})$.

In the Figure 19, there is a comparison between the total power of a wave and an AWLG in same condition. This point has been shown that for the 20 number of airfoil arrangement, the power is approximately same, of course not for very high value of mover velocity (Table 1).

Table 1. A numerical comparison between ALWG and ocean wave energy

\begin{tabular}{|l|l|l|l|l|}
\hline \multicolumn{2}{|c|}{ V =H/2T } & \multirow{2}{*}{ Wave energy (W) } & 20 airfoil (W) & 35 airfoil (W) \\
\cline { 1 - 2 } $\mathrm{H}(\mathrm{m})$ & $\mathrm{V}(\mathrm{m} / \mathrm{s})$ & & & \\
\hline 1 & 0.125 & 1000 & 2000 & 6000 \\
\hline 2 & 0.25 & 8000 & 5000 & 10000 \\
\hline 3 & 0.375 & 15000 & 9000 & 18000 \\
\hline 4 & 0.5 & 30000 & 15000 & 25000 \\
\hline
\end{tabular}



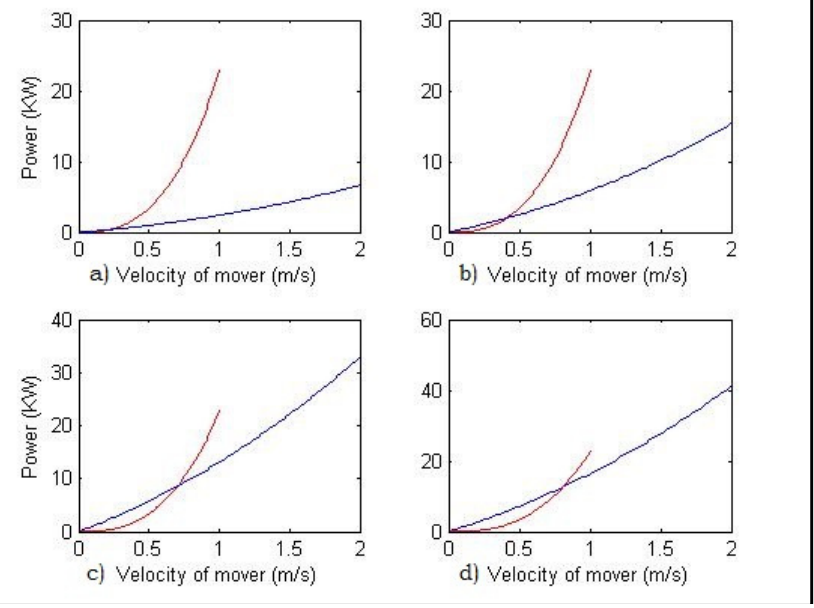

Figure 19. A comparison between the total power of a wave and an AWLG in same condition a) 10 airfoil b) 20 airfoil c) 30 airfoil d) 40 airfoil

\section{Conclusion}

This work has disclosed a study intended to scrutinize airfoil linear wind generator (ALWG) as a new class of wind energy extractor, talented to overcome the main limitations of the present wind energy extractor technologies. Moreover, ALWG has introduced in order to eliminate some of the problems associated with the previous extractor systems such as LWG and ocean-based systems. In fact, ALWG is a new method to produces liner reciprocating motion via attached airfoils to a mover in a linear generator in order to generate electricity.Herein, we have introduced a new mechanism (Figure 5) based on aerodynamic forces exerts on airfoil by air current. In this method we used from linear generator instead of rotary generator. Overall, in our protocol the lock-unlock mechanisms and angle of attack changer is eliminated in order to save energy by use of a flywheel and change of angleof attack only.

Our study shows that in a certain velocity of mover: 1) increasing the projected area of airfoil has a considerable effect on mechanical power and also on induced voltage of generator 2) the mechanical power and induced voltage of generator are increased with addition of initial angle of attack; 3) wind velocity is a contributory factor but its influence is not more higher than increasing projected area and initial angle of attack thus the extraction of more energy from wind is practical using an appropriate design of airfoils in the absence of high wind velocity; 4) the power will be increased dramatically by increasing the number of airfoils.

\section{Acknowledgment}

We gratefully acknowledge Zarand power generation Management Company under management of Mr. Heidary for partial support of this work. Also, we are thankful to Prof. Mansoori and Prof. Ameri in Mechanical Engineering Department of Shahid Bahonar University for their helpful comments. 


\section{References}

[1] Chen D, Xinhui Bi, Jinping Zhao, Laiguo Chen, Jihua Tan, Bixian Mai, Guoying Sheng, Jiamo Fu, Minghung Wong Pollution characterization and diurnal variation of PBDEs in the atmosphere of an E-waste dismantling region, Environmental Pollution, Volume 157, Issue 3, March 2009, Pages 10511057

[2] A reviewontheperformanceofSavoniuswind turbines Renewable and Sustainable Energy Reviews, Volume 16, Issue 5, June 2012, Pages 3054-3064-João Vicente Akwa, Horácio Antonio Vielmo, Adriane Prisco Petry

[3] A review of wave-energy extraction' Johannes Falnes, Marine Structures 20 (2007) 185-201

[4] Energy, exergyandthermo-economicanalysisof solardistillationsystems: A review Renewable and Sustainable Energy Reviews, Volume 27, November 2013, Pages 709-723 K.R. Ranjan, S.C. Kaushik

[5] M.Canale, L. Fagiano,M. Milanese, 'Kitegen: A revolution in wind energy generation '-Journal of energy, November 2008

[6] Ippolito M. Smart control system exploiting the characteristics of generic kites or airfoils to convert energy. European patent \# 02840646, 2004.

[7] Milanese M, Ippolito M. Sistema e procedimento di controllo automatico Del volo di profili alari di Potenza. Patent no. TO2006A000372, 2006 [in Italian]

[8] Determinationof kiteforcesusingthree-dimensionalflight trajectoriesforshippropulsion Renewable Energy, Volume36, Issue10, Octobe, 2011, Pages2667-2678 George M. Dadd, Dominic A. Hudson, R.A. Shenoi

[9] Laddermill, a novel concept to exploit the energy in theairspace Aircraft Design, Volume4, Issues2-3, June-September2001, Pages81-97Wubbo J. Ockel

[10] Historical Aspects of wave energy conversion, Comprehensive Renewable Energy, Volume 8, 2012, pages 7-9, A.F.O. Falcão

[11] Energy harvesting (solar, wind and ocean energy conversion system),Alireza khaligh, OmerC.onar, International Standard Book Number: 978-1-4398-1508-3

[12] Design of a linear generator for wave energy plant, O.Danielsson, Upssala University Sweden

[13] Constantin GHITA,Aurel Iounut CHIRILA,Ioan Dragos DEACONU, and Daniel Ion ILINA,'The magnetizing field of a linear generator used to obtain electrical energy from waves energy 'Department of Electrical Engineering Politehnica University of Bucharest

[14] Linear Electrical actuators and Generators, by I.Boldea (Polytechnic Institute, Timisoara, Romania) and Syed A.Nasar (university of Kentucky)

[15] Energy harvesting (solar, wind and ocean energy conversion system),Alireza khaligh, Omer Conar, International Standard Book Number: 978-1-4398-1508-3

[16] G.T. Heydt, "An assessment of ocean thermal energy conversion as an advanced electric Generation methodology," Proceedings of the IEEE, 81, 409-418, 1993

\section{Authors}

Hossein Darijani received the BSc degree in mechanical engineering from Shahid Bahonar University of Kerman. He received the MSc and $\mathrm{PhD}$, both in mechanical engineering, from Sharif University of Technology (SUT). His technical interestsare Nonlinear Continuum Mechanic Constitutive Modelling of Hyperelastic Materials Non-Linear elasticity Finite Deformation Thermoelasticity Plasticity. Now, he is assistant professor in Shahid Bahonar

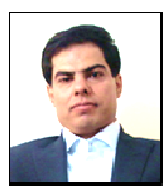
University of Kerman.

Abbas Panahi was born in January 21nd 1992,in Shiraz. He received his BSc degree in mechanical engineering from Shahid Bahonar University of Kerman. His research interests are computational nanotechnology, Atomistic simulation (molecular dynamic), fluid mechanic and renewable energy systems (wind, solar).

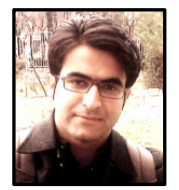

